\title{
Myringoplasty Outcomes From a 5-Year Single Surgeon's Experience and Important Surgical Technical Aspects
}

\author{
Dilhara Karunaratne and Nick Violaris \\ Department of Otolaryngology, Eastbourne District General Hospital, Eastbourne, East Sussex, UK
}

$\begin{array}{ll}\text { Received } & \text { May 15, 2021 } \\ \text { Revised } & \text { June 27, 2021 } \\ \text { Accepted } & \text { July 7, 2021 }\end{array}$

Address for correspondence

Dilhara Karunaratne, BSc (Hons), MBChB (Hons), MRCS, PGCME Department of Otolaryngology,

Eastbourne District General Hospital,

King's Drive, Eastbourne,

BN21 2UD, UK

Tel +44-1323-413704

Fax +44-1323-43579

E-mail d.karunaratne1@nhs.net
Background and Objectives: The United Kingdom (UK) national standard for the closure rate for myringoplasty is $89.5 \%(90.6 \%$ and $84.2 \%$ for primary and revision surgeries, respectively). The average hearing gains for primary and revision myringoplasty are $9.14 \mathrm{~dB}$ and $7.86 \mathrm{~dB}$, respectively. This study compared the myringoplasty outcomes for a single surgeon over 5 years. Subjects and Methods: Data for 68 cases were analyzed retrospectively. The outcome measures were achievement of the tympanic membrane closure and the average hearing gain or loss. Results: The overall and primary closure rates were $97 \%$ and $98 \%$, respectively and significantly higher than the UK national standard $(p=0.0210$ and $p=0.0287$, respectively). The revision closure rate was $93 \%$; however, it was not significantly higher than the national standard $(p=0.1872)$. The average hearing gain was $5.18 \mathrm{~dB}$. The gains for primary and revision surgeries were $5.15 \mathrm{~dB}$ and $5.25 \mathrm{~dB}$, respectively. Conclusions: We propose that these outcomes are a result of our surgical technique, including the simultaneous use of cortical mastoidectomy in ears with discharge. J Audiol Otol 2021;25(4):224-229

Keywords: Tympanic membrane perforation; Tympanoplasty; Mastoidectomy; Outcomes assessment.

\section{Introduction}

Myringoplasty is a surgical operation to graft and repair a persistent perforated tympanic membrane. Common causes for a perforated tympanic membrane are trauma, infection, and tympanostomy tube extrusion. Persistence of the perforation can lead to hearing impairment and chronic otorrhoea. The indications for myringoplasty are to stop chronic otorrhoea, to waterproof the middle ear, and to improve conductive hearing loss. The outcome of myringoplasty success is achievement of an intact tympanic membrane and a dry, self-cleansing ear, with improvements of hearing loss.

With regards to myringoplasty outcomes in the United Kingdom (UK), a 3-year prospective myringoplasty national audit was undertaken and reported in 2015 [1] which evaluated the 3-6 month postoperative outcomes for 33 ENT surgeons and 495 procedures across the UK. The results were that the

This is an Open Access article distributed under the terms of the Creative Commons Attribution Non-Commercial License (https://creativecommons.org/licenses/by-nc/4.0/) which permits unrestricted non-commercial use, distribution, and reproduction in any medium, provided the original work is properly cited. overall closure rate for myringoplasty was $89.5 \%$ (90.6\% for primary surgery and $84.2 \%$ for revision surgery, respectively). The average hearing gains for successful primary and revision myringoplasty were $9.14 \mathrm{~dB}$ and $7.86 \mathrm{~dB}$, respectively.

This study sought to compare the myringoplasty outcomes in terms of achievement of tympanic membrane closure and hearing thresholds for a single surgeon over the period of 5 years against the national standard.

\section{Subjects and Methods}

Permissions were obtained from the audit department at Eastbourne District General Hospital prior to conduction of the study. Ethical approval was not required owing to the retrospective nature of the study and because the study design did not change patient care, treatment, or services. The study was conducted in guidance with the STROBE guidelines for observational studies [2].

\section{Subjects}

In a retrospective fashion, 117 cases of myringoplasty with 
or without ossiculoplasty between 2014 and 2019 under a single surgeon were identified (the 117 cases represented 110 patients, 7 of whom had bilateral surgery done sequentially). Of the 117 cases, 36 cases were excluded due to cholesteatoma, leaving 81 cases suitable for inclusion (these 81 cases represented 74 patients, 7 of whom had bilateral surgery done sequentially). Of the 81 suitable cases, 16 cases (representing 16 patients) were not followed up until at least 3 months, which would limit comparability of closure outcomes to the national standard (where patients were followed up for a minimum of 3 months). All 16 patients were contacted and asked to come to the outpatient department to be re-examined. Three patients attended clinic to be examined and the remaining 13 were excluded from the study. This left 68 cases in total (representing 62 patients, 6 of whom had bilateral surgery done sequentially) to be included in data analysis.

\section{Analysis of surgical results and audiology}

Of the 68 cases, the operation notes, discharge letter, and follow up clinic appointment letters were analyzed, with recording of patient demographics, indication for surgery, whether surgery was primary or revision, surgical technique, choice of graft material, location of the tympanic membrane perforation, and length of follow up.

The first outcome measure was whether closure was achieved or perforation persisted. This was evaluated by checking the follow up clinic letters for documentation of otoscopy findings. As aforementioned, of the patients who were not followed up for up to 3 months, 3 were re-examined and otoscopy findings documented. The second outcome was hearing gain or loss. These were calculated by comparison of preoperative and postoperative audiograms and the averaged air conduction hearing threshold change $(0.5,1,2$, and $5 \mathrm{kHz})$.

\section{Statistical analysis}

Statistical analysis was undertaken by a medical statistician. The test performed was that of a proportion against a hypothesized value and was one sided. Statistical significance was set at $p<0.05$.

\section{Surgical technique}

All cases are done under general anaesthesia and using a microscope. No antibiotics are given on induction. A posterior auricular approach is utilized and temporalis fascia or periosteal graft is harvested (Fig. 1A): 1) The temporalis fascia graft is cleaned of any loose tissue and then left to dry by placing it on a metal gally pot which in turn is placed in a bowl of boiling water (Fig. 1B). 2) Periosteal graft (harvested from the mastoid bone) is used if temporalis fascia has been har- vested previously (usually revision cases) - it is trimmed to thin it as much as possible and then flattened further using a vein press instrument. Access to the ear canal is achieved by making a transverse incision through the meatal soft tissues (halfway down the bony ear canal). The pinna is then held forward by ribbon gauze going through this same incision and self-retaining retractors (Fig. 1C). The edges of the tympanic membrane remnant are freshened. The tympanomeatal flap is raised between 12 o'clock to at least 5 o'clock. Releasing incisions may be needed at the outer ends of the flap at 12 and 6 o'clock to release any tenting of the tympanomeatal flap. The temporalis fascia graft (cut to size) is placed using the underlay technique under the tympanomeatal flap (Fig. 1D). Gel foam is placed within the middle ear to lift up the graft in order to ensure good fit. The tympanomeatal flap is replaced and the ear canal is packed with ribbon gauze soaked in bismuth iodoform paraffin paste (BIPP) - these packs remain in situ for 4 weeks and are removed in outpatient clinic (Fig. 1E). No antibiotics are given postoperatively and patients are typically discharged as a day case. All the cases of ossiculoplasty done in this study were performed by reshaping the patients' own existing bones and did not use prostheses.

\section{Special considerations}

In order to improve the chances of success, several adjustments to the technique may be necessary.

\section{Lifting the drum off the lower $50 \%$ of the handle of the malleus}

This is done in cases where there is a very medialized handle of malleus with no space to place the graft underneath and if the perforation is anterior to the handle of the malleus. This way, we ensure that the graft covers the anterior tympanic membrane perforation fully and is anchored better.

\section{Removal of tympanosclerosis}

Any areas of tympanosclerosis must be removed, especially in the anterior remnant of the drum. This is done gently by using a sharp needle which will lift the superficial layer of the drum off the tympanosclerosis. This will then allow the tympanosclerosis to be dislocated downward and removed.

\section{Kerr flap}

This is done in anterior and very large subtotal perforations with little anterior tympanic membrane remnant. It involves creating a small tunnel anterior to the tympanic membrane annulus using a curved needle, and then by using a small hook, a small amount of the graft is pulled through this tunnel to anchor the graft under the annulus. 

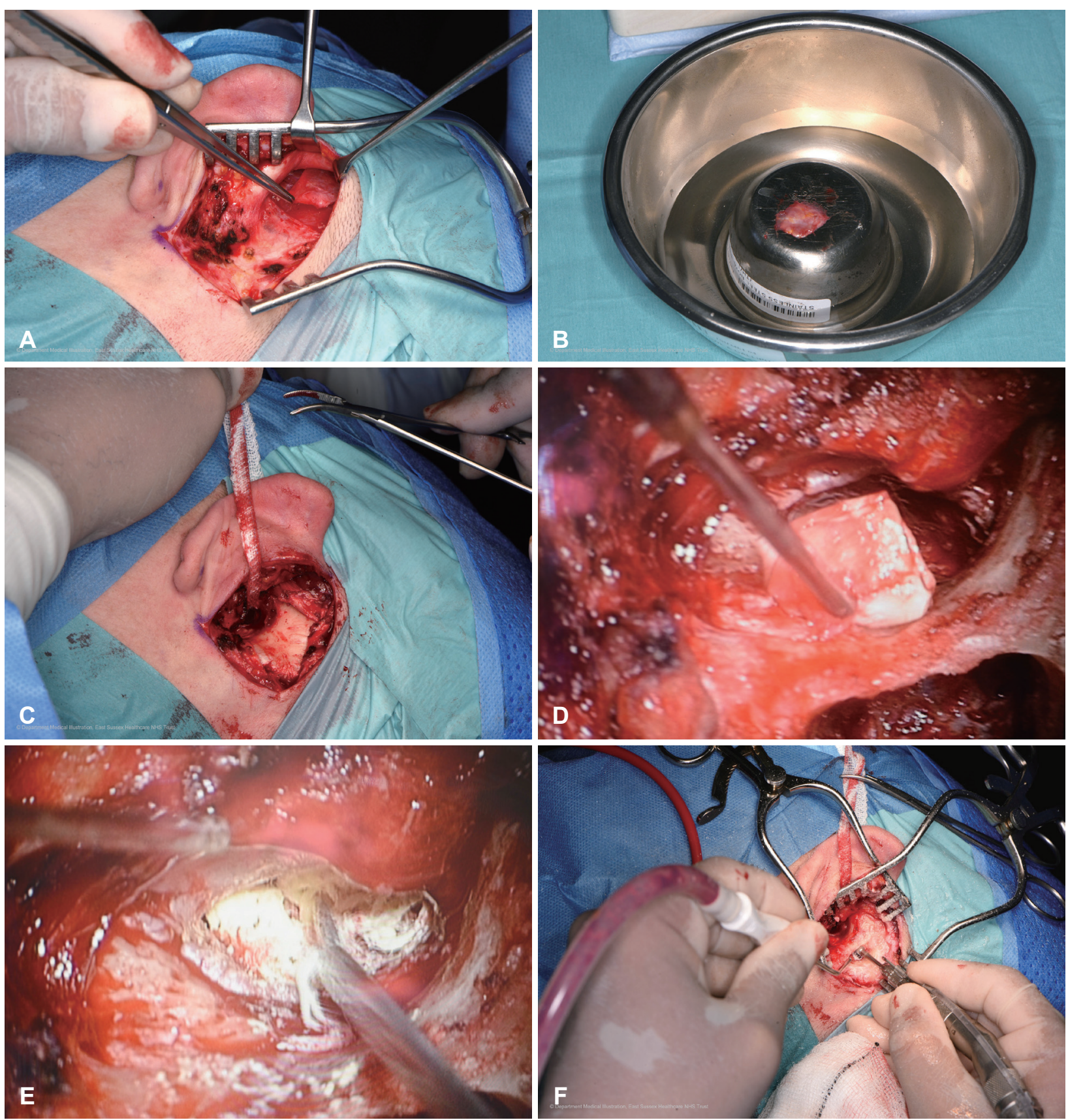

Fig. 1. Surgical technique. A: Posterior auricular incision with harvesting of temporalis fascia. B: Drying of the temporalis fascia by placing it on a metal bowl over hot water. C: The pinna is held forward by ribbon gauze going through a transverse incision through the meatal soft tissues. D: Temporalis fascia graft underlay. E: The canal is packed with ribbon gauze soaked in bismuth iodoform paraffin paste. F: Canal wall up cortical mastoidectomy. All images are left ear.

Lifting the tympanomeatal flap beyond 6 o'clock (sometimes up to 8 o'clock)

By doing this, it enables the graft to be positioned below the inferior annulus in cases where the perforation reaches as far as the inferior annulus of the drum.

Canal wall up cortical mastoidectomy (Fig. 1F)

We feel this is necessary in patients where the ear is con- stantly or intermittently discharging, when the middle ear mucosa is granular or polypoidal and when there are obvious granulations within the middle ear. In these circumstances, the mastoid air cells and antrum invariably will have a similar appearance to the middle ear, suggesting mild underlying osteitis. This must be drilled out to prevent it becoming a future source of infection, resulting in graft failure. 


\section{Small central perforations}

These types of perforations are done with a permeatal approach and fat graft.

\section{Results}

Of the 62 patients included in the study, 33 (53\%) were male and $29(47 \%)$ were female. The left ear was affected in 34 cases and the right ear in 34 cases. There was an age range of 3-81 years (mean \pm SD, 28.35 \pm 20.93 ) (Table 1). Follow up ranged from 3 months to 36 months (mean $\pm \mathrm{SD}, 7.66 \pm 6.54$ ).

With respect to surgical indications, 63 cases $(92.6 \%)$ were due to recurrent infection and 5 cases $(7.4 \%)$ were to stop water precautions. Fifty-four cases $(79.4 \%)$ were primary surgery and 14 cases $(20.6 \%)$ were revision surgery. None of the revision cases were initially operated on by this same surgeon. With regards to surgical procedure performed, myringoplasty alone was used in 24 cases (35.3\%), myringoplasty with ossiculoplasty in 1 case (1.5\%), myringoplasty and cortical mastoidectomy in 40 cases (58.8\%), and myringoplasty with cortical mastoidectomy and ossiculoplasty in 3 cases (4.4\%). Twenty-three (33.8\%) cases had anterior tympanic membrane perforations, 3 (4.4\%) had inferior, 12 (17.6\%) had posterior, 17 (25\%) had central, and 11 (16.2\%) had subtotal. It was not possible to find the perforation type in $2(2.9 \%)$ cases as it was not documented in the notes. The choices of graft material were fat in 1 case $(1.5 \%)$, temporalis fascia in 62 cases $(91.2 \%)$, and periosteum in 5 cases (7.4\%). These results can be seen collated in Table 2 .

The overall closure rate was $97 \%(66 / 68)$ and significantly higher than the national standard $(p=0.0210)$. The primary closure rate was $98 \%$ (53/54) and significantly higher than the national standard $(p=0.0287)$. The revision closure rate was $93 \%(13 / 14)$ but not significantly higher than the national standard ( $p=0.1972)$.

In calculation of hearing thresholds, 2 cases were excluded due to non-intact tympanic membrane and a further 5 cases were excluded as no audiogram was available and it was not possible to arrange a further audiology appointment with the patients in question. The ossiculoplasty patients were within these 7 excluded cases and therefore did not bias the hearing results.

Table 1. Age distribution of the patients

\begin{tabular}{cc}
\hline Age rage $(\mathrm{yr})$ & Cases $(\%)$ \\
\hline $3-6$ & $3(4.4)$ \\
$7-10$ & $11(16.2)$ \\
$11-14$ & $11(16.2)$ \\
$15-18$ & $8(11.8)$ \\
$>18$ & $35(51.5)$ \\
\hline
\end{tabular}

An average gain of $5.18 \mathrm{~dB}$ was calculated for the remaining cases. This can be seen in Table 3. From this it can be seen that the average gain for primary surgery is $5.15 \mathrm{~dB}$ and for revision surgery is $5.25 \mathrm{~dB}$. Since the national standard for gains are given as $9.14 \mathrm{~dB}$ for primary surgery and $7.86 \mathrm{~dB}$ for revision surgery, there is no point in formally testing to see whether our gains are superior.

With regards to postoperative complications, there was documented evidence of 2 patients having posterior auricular wound infection and 1 patient having a temporary taste disturbance that resolved within 6 months.

\section{Discussion}

Myringoplasty is a common procedure within otolaryngo-

Table 2. Analysis of myringoplasty outcomes and technique from the 68 cases

\begin{tabular}{lc}
\hline \multicolumn{1}{c}{ Case characteristics } & $\begin{array}{c}\text { Number of } \\
\text { cases }(\mathrm{n}=68)\end{array}$ \\
\hline Surgical indication & \\
Recurrent infections & $63(92.6)$ \\
Waterproofing & $5(7.4)$ \\
Perforation type ( $\mathrm{n}=66)^{*}$ & \\
Anterior & $23(33.8)$ \\
Central & $17(25.0)$ \\
Posterior & $12(17.6)$ \\
Subtotal & $11(16.2)$ \\
Inferior & $3(4.4)$ \\
Surgery & \\
Primary & $54(79.4)$ \\
Revision & $14(20.6)$ \\
Surgical procedure & \\
Myringoplasty and cortical mastoidectomy & $40(58.8)$ \\
Myringoplasty alone & $24(35.3)$ \\
Myringoplasty, ossiculoplasty, & $3(4.4)$ \\
cortical mastoidectomy & \\
Myringoplasty and ossiculoplasty & $1(1.5)$ \\
Choice of graft material & \\
Temporalis fascia & $62(91.2)$ \\
Periosteum & $5(7.4)$ \\
\hline Fat & $1(1.5)$ \\
\hline
\end{tabular}

Data are presented as $\mathrm{n}(\%)$. Percentages have been rounded and may not total to $100 \%$. *Two cases were not documented.

Table 3. Post operative hearing gains

\begin{tabular}{lrcc}
\hline Surgery & Total gains $(\mathrm{dB})$ & Cases $(\mathrm{n})$ & $\begin{array}{c}\text { Average gain }(\mathrm{dB}), \\
\text { mean } \pm \text { SD }\end{array}$ \\
\hline Primary & 247.43 & 48 & $5.15 \pm 15.11$ \\
Revision & 68.30 & 13 & $5.25 \pm 12.00$ \\
Overall & 315.73 & 61 & $5.18 \pm 14.41$ \\
\hline
\end{tabular}


logical practice, but outcomes differ and there is much variation in surgical technique and practice [3]. Even within the UK national audit, this variation in practice is not explored [1]. It is noted in the literature that multiple factors influence the success of myringoplasty in achieving tympanic membrane closure with patient age, site and size of the perforation, type of graft, surgical technique, the surgeon's experience, and the condition of the ear all playing a role [4]. At present there is no clear consensus on the optimal tympanoplasty technique [3].

The operation can be done microscopically or endoscopically. There are three main approaches used in the access of the tympanic membrane for myringoplasty [5] - endaural, posterior auricular, and permeatal. The surgeon's preferences and the site of the perforation determines the approach. The endaural approach is usually for posterior or central perforations, the posterior auricular for anterior perforations, and the permeatal for central perforations [5]. Perforation edges must be freshened to facilitate healing, and removal of tympanosclerosis adjacent to the tympanic membrane perforation is an essential part of the success of our surgical technique, as its remainder to shown to result in poorer outcomes [6].

There are many choices of graft to use in the reconstruction of the tympanic membrane such as cartilage, fat, perichondrium, periosteum, vein, or temporalis fascia [7]. Our personal preference is temporalis fascia for primary cases or periosteum in revision cases (as temporalis fascia may have already been harvested in these cases). Due to this, our surgical incision is posterior auricular, which facilitates harvesting of both temporalis fascia and periosteum. Although some studies show the supposed superiority of cartilage grafts versus temporalis fascia [8], our results demonstrate the value and success of the technique using temporalis fascia.

There are two main approaches to positioning of the graft in the reconstruction of the tympanic membrane: the underlay and overlay techniques. The underlay technique positions the graft medial to the tympanic membrane remnant and to the handle of the malleus. It thought to be more suited to posterior perforation and technically easier to perform [9]. The overlay technique is thought to be more suitable for anterior and subtotal perforations, but technically challenging [10]. The underlay technique utilizing temporalis fascia is the commonest technique used [11]. Studies demonstrate that the underlay technique is associated with more effective graft uptake and less complications [12]. It is our practice to use the underlay technique exclusively and this may be partially responsible for our favorable outcomes. Our modification to the underlay technique includes the passing the graft lateral to the handle of the malleus (but medial to the tympanic membrane) in cas- es where the handle of the malleus is extremely medialized and in anterior or antero-superior perforations. Positioning the graft this way ensures a tighter fit of the graft around the tympanic membrane perforation and reduces risk of migration, which therefore promotes improved healing. We feel that this simple technique improves the success of surgery in these types of perforations.

The Kerr flap is a technique we use to close anterior and near total perforations. These particular types of perforations are challenging to close because of the lack of anterior support for the graft, with studies demonstrating that anterior perforations in particular have poorer closure rates and hearing outcomes [5]. The Kerr flap involves quilting the graft through an anterior tunnel, which increases graft stability by preventing retraction [13], with studies demonstrating its effectiveness in achieving perforation closure and positively influencing hearing gain [13]. The majority of the perforation types within our study were anterior (23 cases), and the Kerr flap was used in all of them, with only two cases (one primary and one revision) having failed closure at follow up.

A further variant of our technique is the extent of lifting of the tympanomeatal flap. We recommend lifting the inferior fibrous annulus well beyond 6 o'clock (up to 8 o'clock if necessary). This allows the graft to be placed beneath the inferior extent of the perforation, ensuring better fit and preventing displacement, thus preventing failure of closure of the perforation. All of our inferior perforation cases were successfully closed utilizing this technique.

Some surgeons opt to perform simultaneous cortical mastoidectomy in discharging ears, as it is recognised that poor pneumatization of the mastoid predisposes to chronic suppurative otitis media. However, the evidence regarding the benefit of this additional procedure is conflicting, with some being of the opinion that mastoidectomy is the best way of aerating the mastoid and removing sources of infection within the air cells $[14,15]$ and other studies suggesting that there is no demonstrable difference in outcomes whether or not it was performed [16]. Our observations are that simultaneous canal wall up cortical mastoidectomy in discharging ears improves graft uptake outcomes as it eliminates residual sources of infection and we also demonstrate no significant patient complications as a result of this added procedure.

Our study demonstrates that our surgical technique achieves excellent outcomes that are statistically significantly better than the national standard in the UK. Strengths of the study are the longer term follow up of patients (mean 7.66 months) with respect to the UK myringoplasty paper (3-6 month follow up), which may suggest that our surgical technique may result in better longer-term achievement of tympanic mem- 
brane closure. However, it is increasingly being recognized that re-perforation may occur many years after the surgery [5], but such long-term follow up is not always feasible.

One of the potential drawbacks to this technique, based upon our results, is that the hearing improvements are not as high as the national standard. We believe that this may be in part due to the audiograms being done early in the stage of healing (the majority were done at 4 weeks when the patient had their first follow up to remove the BIPP pack in the ear canal), and so we hypothesize that hearing may have improved further down the course of recovery. It has not been possible to retest the hearing thresholds of the patients again due to a combination of factors, including coronavirus restrictions, patient unavailability, and the resource constraints of the UK National Health Service.

Further limitations of this study are its retrospective design which led to exclusion of some patients due to missing notes and also that the notes did not document the size of the tympanic membrane perforation, which is known to correlate with surgical success (larger perforations have less surgical success) [17]. However, it can be noted that $16.2 \%$ of cases within our series were subtotal perforations, all of which achieved successful closure.

There is also the possibility that the results may correlate with the experience of the surgeon (30 years of surgical experience), therefore reproducibility of results by less experienced surgeons may not be achieved. Despite this, the study adds an easily adoptable surgical technique in the literature for otologists to trial. It also provides support for the simultaneous use of cortical mastoidectomy and demonstrates that good outcomes can be achieved with temporalis fascia graft underlay.

In conclusion, this surgical technique can be easily adopted, used for all types of tympanic membrane perforations and gives good results of graft uptake, and results in hearing improvement with no significant complications. This study adds to the literature support for use of cortical mastoidectomy in discharging ears, demonstrates that temporalis fascia graft can be used to achieve outcomes comparable to other types of graft, and shows that the underlay technique leads to favourable outcomes.

\section{Acknowledgments}

Dr. Gwyn Jones who was responsible for the analysis of the data. Mr. Ravi Nokku (paediatric audiologist) and Mrs. Karen Brett (senior audiology administrator) both of whom were of enormous help in obtaining audiograms. Mrs. Ciara Pooley (theatre administrator) who helped provide a list of patients who underwent myringoplasty during the specified time period.

\section{Conflicts of interest}

The authors have no financial conflicts of interest.

\section{Author Contributions}

Conceptualisation: Nick Violaris. Data curation: Dilhara Karunaratne. Formal analysis: Dilhara Karunaratne. Investigation: Dilhara Karunaratne. Methodology: Dilhara Karunaratne. Project administration: Nick Violaris. Resources: Dilhara Karunaratne. Software: Dilhara Karunaratne. Supervision: Nick Violaris. Validation: Dilhara Karunaratne. Visualization: Dilhara Karunaratne. Writing — original draft: Dilhara Karunaratne. Writing — review \& editing: Nick Violaris. Approval of final manuscript: all authors.

\section{ORCID iD}

Dilhara Karunaratne https://orcid.org/0000-0003-0705-7176

\section{REFERENCES}

1) Phillips JS, Yung MW, Nunney I. Myringoplasty outcomes in the UK. J Laryngol Otol 2015;129:860-4.

2) von Elm E, Altman DG, Egger M, Pocock SJ, Gøtzsche PC, Vandenbroucke JP. The Strengthening the Reporting of Observational Studies in Epidemiology (STROBE) statement: guidelines for reporting observational studies. Ann Intern Med 2007;147:573-7.

3) Chidlow C, Prunty S, Hinton-Bayre A, Shubhraj S, Renton J, Kuthubutheen J. Practice patterns in myringoplasty surgery among Australian surgeons. Aust J Otolaryngol 2020;3:28

4) Hirsch BE. Myringoplasty and tympanoplasty. In: Operative Otolaryngology: Head and Neck Surgery (ed. Myers EN), 2nd ed. Philadelphia: WB Saunders;2008. p.1133-45.

5) Aggarwal R, Saeed SR, Green KJ. Myringoplasty. J Laryngol Otol 2006;120:429-32.

6) Salvador P, Gomes P, Silva F, Fonseca R. Type I tympanoplasty: surgical success and prognostic factors. Acta Otorrinolaringol Esp (Engl Ed) 2021;72:182-9.

7) Patil K, Baisakhiya N, Deshmukh PT. Evaluation of different graft material in type 1 tympanoplasty. Indian J Otol 2014;20:106.

8) Odat H, Alali M, Kanaan Y, Al-Qudah M. Success rate of type 1 tympanoplasty: a comparative study. J Laryngol Otol 2021;135:315-9.

9) Kulduk E, Dundar R, Soy FK, Guler OK, Yukkaldiran A, Iynen I, et al. Treatment of large tympanic membrane perforations: medial to malleus versus lateral to malleus. Indian J Otolaryngol Head Neck Surg 2015;67:173-9.

10) Gersdorff M, Gérard JM, Thill MP. Overlay versus underlay tympanoplasty. Comparative study of 122 cases. Rev Laryngol Otol Rhinol (Bord) 2003;124:15-22.

11) de Savornin Lohman EAJ, Borgstein J. Transmeatal tympanoplasty of subtotal and anterior perforations: a single-institution experience including 94 patients. Clin Otolaryngol 2017;42:920-3.

12) Khawaja M, Sajid T, Aziz T, Ashfaq U, Khan A. Comparison of graft uptake by underlay and overlay technique in myringoplasty. J Ayub Med Coll Abbottabad 2020;32(Suppl 1):S640-3.

13) Sharp JF, Terzis TF, Robinson J. Myringoplasty for the anterior perforation: experience with the Kerr flap. J Laryngol Otol 1992;106:14-6.

14) Tos M. Manual of middle ear surgery. 1st ed. New York: Thieme;1993. p.5.

15) McGrew BM, Jackson CG, Glasscock ME 3rd. Impact of mastoidectomy on simple tympanic membrane perforation repair. Laryngoscope 2004;114:506-11.

16) Albu S, Trabalzini F, Amadori M. Usefulness of cortical mastoidectomy in myringoplasty. Otol Neurotol 2012;33:604-9.

17) Vaidya S, Sharma JK, Singh G. Study of outcome of tympanoplasties in relation to size and site of tympanic membrane perforation. Indian J Otolaryngol Head Neck Surg 2014;66:341-6. 